Article

\title{
Transcriptional Regulation of gga-miR-451 by AhR:Arnt in Mycoplasma gallisepticum (HS Strain) Infection
}

\author{
Yabo Zhao, Yali Fu, Yingfei Sun, Mengyun Zou and Xiuli Peng * \\ Key Laboratory of Agricultural Animal Genetics, Breeding and Reproduction, Ministry of Education; \\ College of Animal Science and Technology and College of Veterinary Medicine, Huazhong Agricultural \\ University, Wuhan 430070, China; zyb@webmail.hzau.edu.cn (Y.Z.); fuyali@webmail.hzau.edu.cn (Y.F.); \\ sunyingfei@webmail.hzau.edu.cn (Y.S.); zoumengyun@webmail.hzau.edu.cn (M.Z.) \\ * Correspondence: xlpengsishun@mail.hzau.edu.cn; Tel.: +86-27-87281396; Fax: +86-27-87280408
}

Received: 30 April 2019; Accepted: 21 June 2019; Published: 24 June 2019

\begin{abstract}
MicroRNAs (miRNAs) have been determined to be important regulators for pathogenic microorganism infection. However, it is largely unclear how miRNAs are triggered during pathogen infection. We previously reported that the up-regulation of gga-miR-451 negatively regulates the Mycoplasma gallisepticum (MG)-induced production of inflammatory cytokines via targeting tyrosine3-monooxygenase/tryptophan5-monooxygenase activation protein zeta (YWHAZ). The aim of this study was to investigate the mechanism regulating gga-miR-451 in MG infection in chickens. Analysis of gga-miR-451 precursor, pri-miR-451, and pre-miR-451 indicated that the regulation occurred transcriptionally. We also identified the transcriptional regulatory region of gga-miR-451 that contained consensus-binding motif for aryl hydrocarbon receptor (AhR) and aryl hydrocarbon receptor nuclear translocator (Arnt) complex, which is known as the transcription factor that regulates gene expression. Luciferase reporter assays combined with chromatin immunoprecipitation (ChIP) demonstrated that AhR:Arnt bound directly to the promoter elements of gga-miR-451, which were responsible for gga-miR-451 transcription in the context of MG infection. Furthermore, upregulation of AhR:Arnt significantly induced gga-miR-451 and inhibited YWHAZ expression, suggesting that AhR:Arnt may play an anti-inflammatory role in MG infection. This discovery suggests that induced gga-miR-451 expression is modulated by AhR:Arnt in response to MG infection.
\end{abstract}

Keywords: AhR:Arnt; gga-miR-451; Mycoplasma gallisepticum HS strain; YWHAZ

\section{Introduction}

MicroRNAs (miRNAs) are composed of a large class of post-transcriptional regulators of gene expression, and the majority of miRNAs are originally transcribed in the nucleus RNA polymerase II. Following transcription, the primary transcripts of miRNAs (pri-miRNAs) are processed by RNAase III enzyme, Drosha, into 65-100 nucleotide (nt) precursor miRNAs (pre-miRNAs) to be transported into the cytoplasm and cleaved by the RNAase III, Dicer, to give rise to double-stranded, mature miRNAs ( 22 nt). The miRNA passenger strand is degraded while the miRNA guide strand is loaded into an Argonaute protein to form the RNA-induced silencing complex (RISC). The RISC then produces mature miRNA, which enables partial base pairing and negative regulation of protein synthesis and/or mRNA degradation in the majority of cases [1]. In chickens, miRNAs have been reported to regulate diverse developmental, physiological, and pathological processes including avian diseases [2]. For example, gga-miR-19a activates the NF- $\kappa B$ signaling pathway through targeting ZMYND11 and promotes the proliferation of chicken fibroblast (DF-1) cells upon Mycoplasma gallisepticum HS strain (MG-HS) infection [3]. gga-miR-23b promotes the replication of Avian Leukosis virus by repressing 
the expression of IRF1 [4]. gga-miR-375 inhibits cell proliferation in tumorigenesis post subgroup J avian leukosis virus infection [5]. Although the function of host miRNAs has been generally studied during various foreign pathogen infections, there is very limited knowledge on how the expression of miRNAs is triggered during these diseases.

Approximately $50 \%-70 \%$ of miRNAs are located in intergenic sites or in antisense orientation to annotated genes with independent transcription units. While the remaining miRNAs are encoded within the introns of protein coding genes, they might be transcribed in parallel with host transcripts and as part of the host genes [6]. Different mechanisms of biogenesis are required depending on the two different transcription classes of miRNA. Previous studies reported that the expression of miRNAs was generally regulated by transcriptional factors and co-regulators. For instance, the transcription factor Ppara directly binds the promoter region of Hsa-miR-181a2 and up-regulates its expression [7]. In mouse granulosa cells, transcription factor CP2 enhances the expression of miR-144 [8]. Several miRNAs, including let-7, miR-21, miR-146a, miR-155, miR-181, and the miR-17-92 cluster have been demonstrated as targets of the $N F-\kappa B$ transcription factors [9].

Recently, chronic respiratory disease (CRD) of chickens and turkeys has emerged as a great challenge to poultry farmers and creates a large financial burden for poultry industries around the world. The major causative agent is Mycoplasma gallisepticum (MG), which is a bacterium belonging to the class Mollicutes and the family Mycoplasmataceae and has the ability to penetrate chicken fibroblasts and HeLa cells to establish infection [10]. During infection, MG integrates with host respiratory epithelial cells (trachea, lungs, and air sacs) and causes local or systemic inflammatory response [11]. We previously reported that gga-miR-101, gga-miR-19a, and gga-miR-451 were up-regulated in both MG-infected DF-1 cells and MG-infected chicken embryonic lungs to modulate MG-triggered inflammation by targeting genes involved in inflammatory signal pathways $[3,12,13]$. However, the knowledge of the mechanisms for how miRNA genes themselves are triggered has comparatively lagged behind.

Our previous study indicated the property of gga-miR-451 as an anti-inflammatory miRNA, which negatively regulates the MG-induced production of inflammatory cytokines through targeting YWHAZ [13]. In mammals, miR-451 has also been reported to play a critical role in regulating inflammation in influenza-infected cells [14]. To further explain the effects of gga-miR-451 in MG-HS infection, it will be crucial to understand how gga-miR-451 gene is up-regulated. In this study, we found that the pri-miR-451 and pre-miR-451 were up-regulated in MG-HS infection, suggesting that regulation of gga-miR-451 occurs transcriptionally. Promoter analysis revealed that AhR:Arnt plays an important role in induction of gga-miR-451 transcription upon MG-HS infection. Furthermore, we found that AhR:Arnt was significantly up-regulated upon MG-infected DF-1 cells and was a negative regulator of YWHAZ expression. To our knowledge, this is the first time that the transcriptional regulation of gga-miR-451 was identified.

\section{Results}

\subsection{Primary Transcript of gga-miR-451 Is Induced in MG-infected DF-1 Cells}

Our previous data show that gga-miR-451 level was significantly up-regulated in both MG-infected DF-1 cells and MG-infected chicken embryonic lungs [13]. To determine the kinetics of primary gga-miR-451 transcript (pri-miR-451) and gga-miR-451 precursor (pre-miR-451) after MG infection, DF-1 cells were infected with MG-HS for the indicated times, with non-infected acting as the control group. As shown in Figure 1A, pMGA1.2, the adhesion protein from MG-HS, was highly expressed at 24,48 , and $72 \mathrm{~h}$ post-infection (hpi). We further measured the expression of pri-miR-451 and pre-miR-451, and they were up-regulated with no time-dependent increase in cells following MG infection (Figure 1B,C). These data suggest that the upregulation of gga-miR-451 may happen at the transcriptional level. 
A

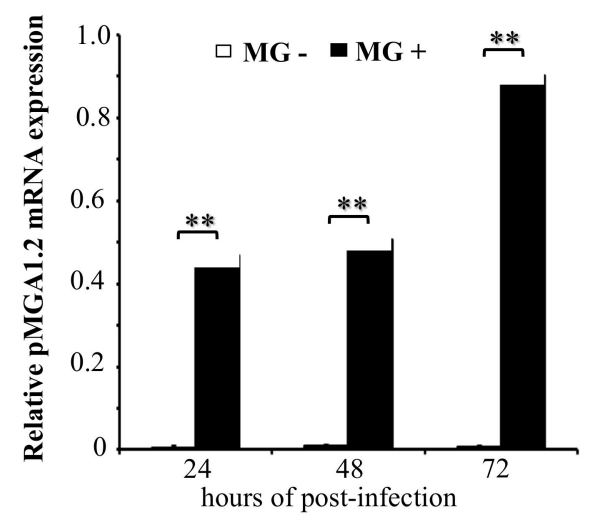

B

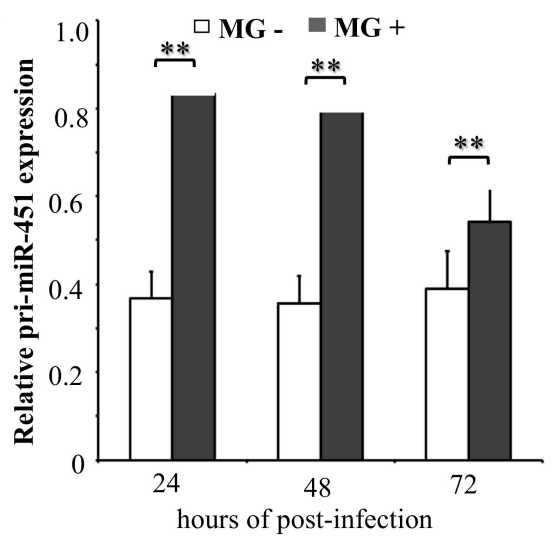

$\mathrm{C}$

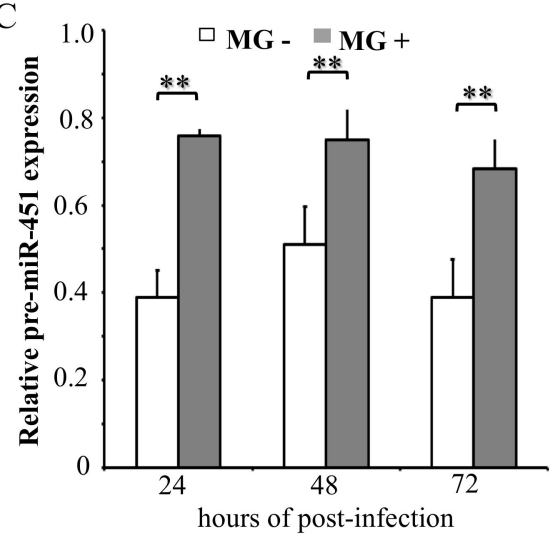

Figure 1. Differential expression of primary transcripts of Mycoplasma gallisepticum (MG)-induced gga-miR-451. The total RNA was extracted at 24,48 , and $72 \mathrm{~h}$ post-infection (hpi), and the expression of pMGA1.2 (A), pri-miR-451 (B), and pre-miR-451 (C) were assessed by RT-qPCR normalizing to the expression of glyceraldehyde-3-phosphate dehydrogenase (GAPDH) in the samples. Three independent experiments, each with three replicates, were performed. Student's $t$ test was used to analyze the significant differences. The plotted data points show the means \pm SDs, and the asterisks indicate statistically significant differences $\left({ }^{* *} p<0.01\right)$.

\subsection{Identification of the Promoter Region for gga-miR-451}

The detailed mechanism of how the MG-HS infection triggered the expression of pri-miR-451 was studied. The location and genomic information of gga-miR-451 that were acquired from NCBI (https://www.ncbi.nlm.nih.gov/gene/777915) showed that gga-miR-451 resides on chromosome 19 and between ERAL1 gene and FLOT2 gene. To examine the transcriptional regulation of gga-miR-451 in MG-infected DF-1 cells, we tried to determine the promoter region of gga-miR-451. To identify the range of the minimal promoter region (PR), an upstream region of about 2.2-kb DNA fragment was selected for promoter mapping, followed by a series of deletions of this DNA fragment and construction into luciferase reporter plasmids (pGL-3). These reporter plasmids were separately transfected into DF-1 cells to determine the basal and MG-induced promoter activities. The luciferase reporter assay indicated that full-length reporter (PR-2174) had explicit MG inducibility, which was reduced by three cuttings of this full-length region, PR-1612, PR-1147, and PR-542 (Figure 2). However, the reporter PR-386 had a higher luciferase activity in both non-infected DF-1 cells and MG-infected DF-1 cells, compared to the reporter PR-254, PR-177, suggesting that PR-386 ( -386 bp to -254 bp, $132 \mathrm{bp}$ ) is required for gga-miR-451 transcriptional activity and possesses fully intact promoter activity. 


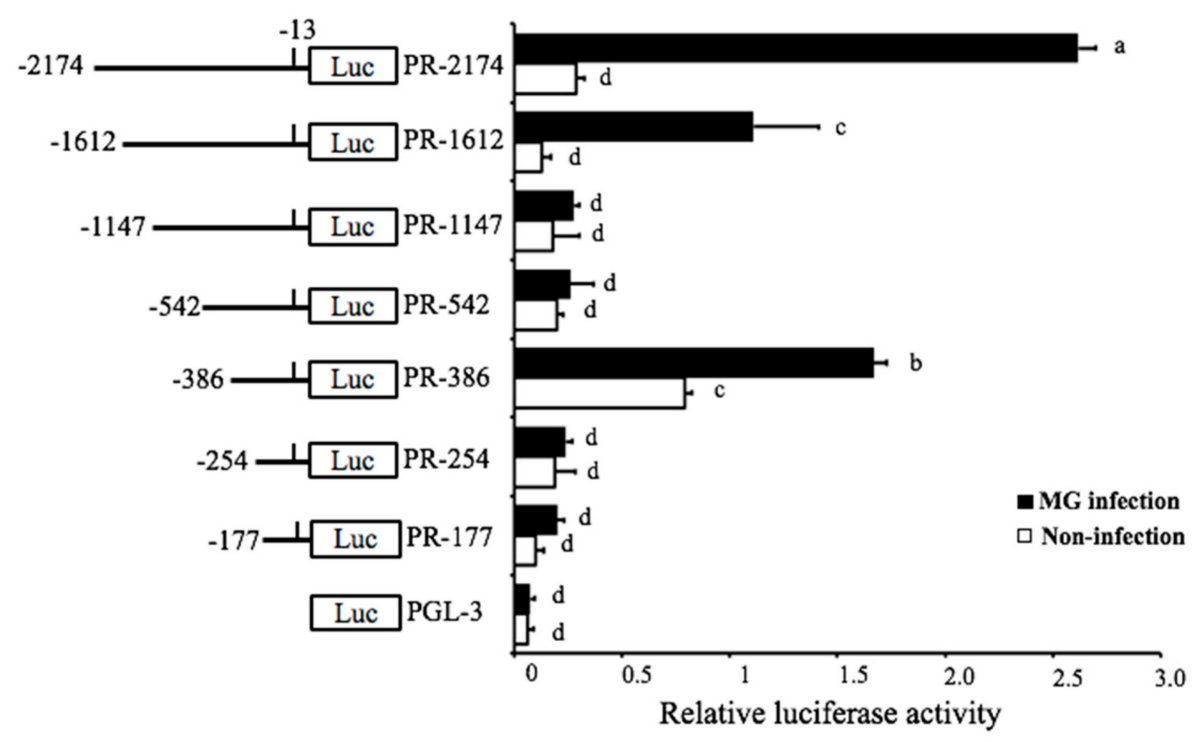

Figure 2. Identification of the boundary of gga-miR-451 promoter region. Chicken fibroblast (DF-1) cells were co-transfected with promoter vectors $(100 \mathrm{ng})$ of different length of the gga-miR-451 promoter region and PRL-TK (10 ng) for $12 \mathrm{~h}$ and then either left uninfected or infected with $30 \mu \mathrm{L}$ of MG-HS, At $24 \mathrm{hpi}$, samples were collected and the luciferase activity was measured by a dual-luciferase glow assay; the firefly luciferase activity was standardized to Renilla luciferase activity, and pGL3 was used as control. The data are expressed as the means \pm SDs. Different lowercase letters represent $p<0.01$, determined by ANOVA.

\subsection{AhR:Arnt Binding Sites are Necessary for gga-miR-451 Promoter Activity}

To further decipher the transcription factors binding to the core promoter of gga-miR-451, we used the transcription factor prediction software from gene-regulation (http://gene-regulation.com) and JASPAR (http://jaspar.genereg.net) to analyze the putative binding sequences ( $-386 \mathrm{bp}$ to $-254 \mathrm{bp}$ ), the potential transcription factors, including AREB6, AhR:Arnt, Barbie Box, and v-Maf predicted within gga-miR-451 promoter (Figure S1). The transcription factor-binding sites (TFBS) were also identified in the PR-386 region (132bp) by the prediction software with three binding sites by AhR:Arnt (Figure 3A). To detect if the predicted transcription factors were responsive to gga-miR-451 transcription, we constructed dual-luciferase reporter plasmids carrying a series of truncated promoters, including full-length (F), truncation 1 (T1-374bp), T2-345bp, T3-331bp, and T4-319bp (Figure 3B). The luciferase activity assay showed that deletion of AREB6 binding site did not have significant effect on luciferase activity compared to the full-length promoter construction. However, when AhR:Arnt binding site was truncated, the MG-induced luciferase activity was significantly decreased (Figure 3B), suggesting AhR:Arnt binding site was necessary for gga-miR-451 promoter activation. Furthermore, deletion of Barbie Box and v-Maf binding sites had no significant effect on luciferase activity compared to the deletion of AhR:Arnt binding site. To further clarify whether the three AhR:Arnt binding sites were all core sites, seven different dual-luciferase reporter plasmids including mutant 1 (Mut1), Mut2, Mut3, mutants 1 and 2 (Mut12), Mut13, Mut23, and Mut123, were constructed. As shown in Figure 3C, mutant123 significantly affected MG-induced luciferase activity compared to the full-length promoter construction, whereas the other different mutants did not affect non-infection luciferase activity but reduced MG-induced promoter activity. These results indicate that the three AhR:Arnt binding sites are necessary for the activation of gga-miR-451 promoter by MG infection (Figure 3D). 

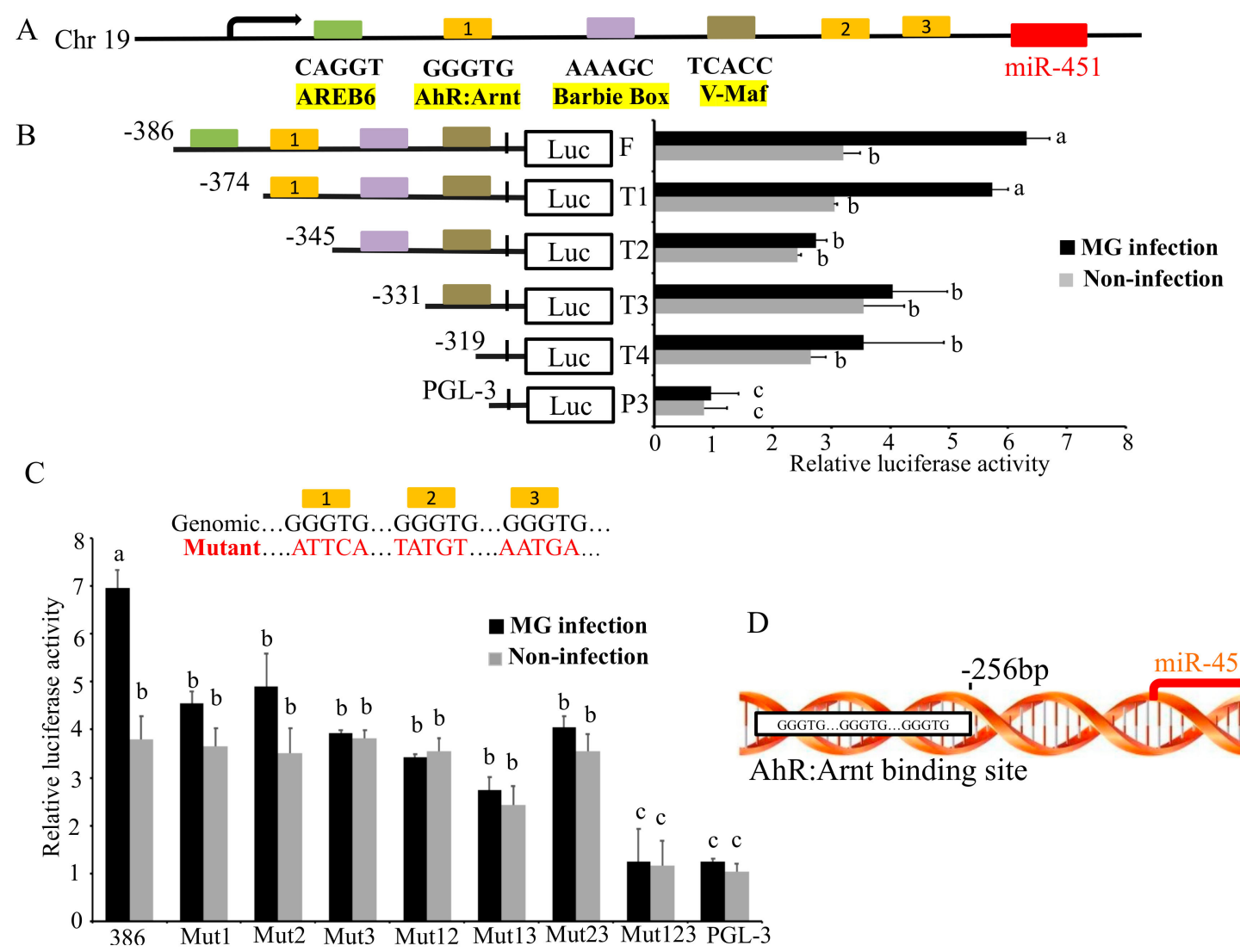

Figure 3. aryl hydrocarbon receptor (AhR):aryl hydrocarbon receptor nuclear translocator (Arnt) regulates gga-miR-451 promoter activity. (A) Schematic diagram of gga-miR-451 genomic loci on chicken chromosomes 19. Potential binding sites of AREB6, AhR:Arnt, Barbie Box, and v-Maf transcriptional factors (TFs) are shown as different color boxes; the sequences are under the boxes. (B) Schematic representation of truncation T1 to T4 in the full-length gga-miR-451 promoter. DF-1 cells were co-transfected with promoter vectors (100 ng) with different deletions and PRL-TK (10 ng) for $12 \mathrm{~h}$ and then either uninfected or infected with $30 \mu \mathrm{L}$ of MG-HS. At $24 \mathrm{~h}$ post-infection, both firefly and renilla luciferase activity were measured through a dual-luciferase reported assay. (C) Schematic representation of different point mutations (Mut1, 2, and 3) in the wild-type promoter (386). The details of dual-luciferase reported assay are the same as above. (D) Sequence schematic diagram of AhR:Arnt binding site in the promoter region of gga-miR-451. The data are shown as means \pm SDs. Different lowercase letters represent $p<0.01$, determined by ANOVA.

\subsection{AhR:Arnt Enhances the Transcriptional Activity of the gga-miR-451 Promoter}

To verify the role that AhR:Arnt plays in MG-induced activation of gga-miR-451 promoter, the effects of AhR:Arnt on the transcriptional activity of the gga-miR-451 promoter were evaluated by over-expressing AhR:Arnt with pcDNA3.1 vector and silencing AhR:Arnt with siRNA. pcDNA3.1-AhR (p-AhR) and pcDNA3.1-Arnt (p-Arnt) could substantially increase AhR and Arnt expression. The siRNA-AhR:Arnt (si-AhR:Arnt) could significantly inhibit AhR:Arnt expression in DF-1 cells (Figure S2). The p-AhR, p-Arnt, pcDNA3.1-AhR:Arnt (p-AhR:Arnt), control pcDNA3.1, si-AhR:Arnt, or a nonspecific control siRNA (si-NC) was co-transfected with the pGL3-386 vector into DF-1 cells, and then either left uninfected or infected with MG at $24 \mathrm{hpi}$, followed by luciferase reporter assays. As shown in Figure 4A,B, overexpression of AhR:Arnt together significantly increased MG-induced gga-miR-451 promoter activity. However, overexpression of AhR or Arnt on their own did not affect the luciferase activity. In contrast, knockdown of AhR:Arnt decreased gga-miR-451 promoter activity. These results indicate that AhR:Arnt regulates gga-miR-451 promoter activity. 
A

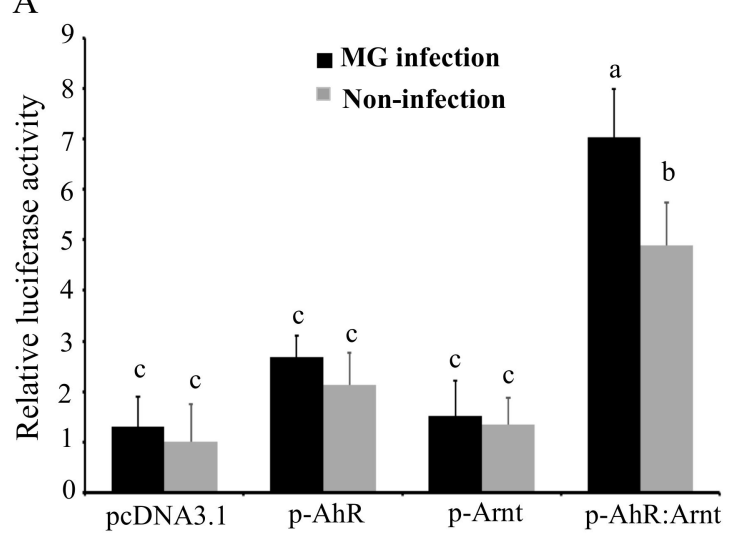

B

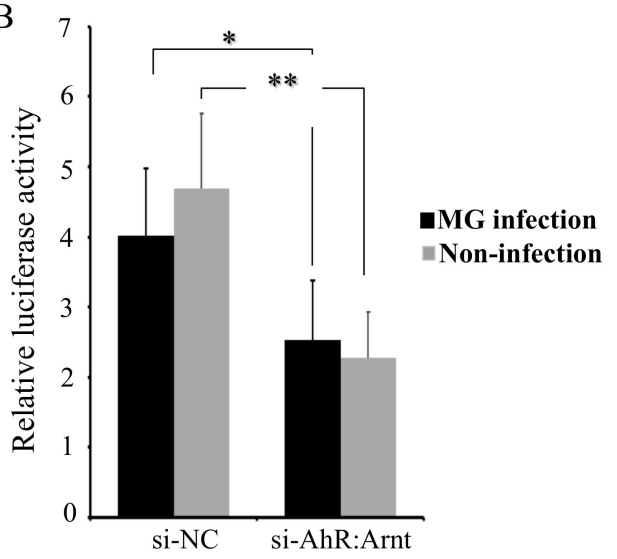

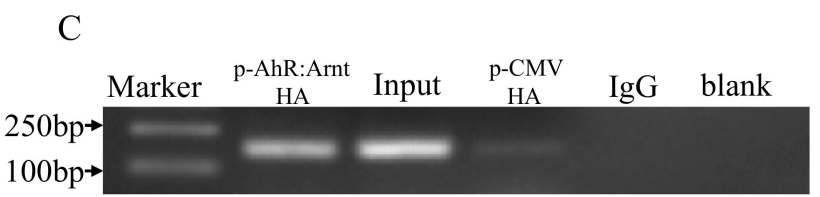

Figure 4. AhR:Arnt directly binds to gga-miR-451 promoter. (A) DF-1 cells were co-transfected with pGL3-386 vector (100 ng), PRL-TK vector (10 ng), and pcDNA3.1, p-AhR, p-Arnt, or p-AhR:Arnt (50 ng AhR and $50 \mathrm{ng}$ Arnt) for $12 \mathrm{~h}$, then either left uninfected or infected with MG. At $24 \mathrm{hpi}$, luciferase reporter assays detected the gga-miR-451 promoter activities. (B) DF-1 cells were co-transfected with pGL3-386 vector, PRL-TK vector, and si-NC, si-AhR:Arnt for $12 \mathrm{~h}$, then either left uninfected or infected with MG-HS. At $24 \mathrm{hpi}$, luciferase reporter assays detected the gga-miR-451 promoter activities. (C) ChIP-qPCR assay was performed using chromatin isolated from pCMV-C-HA-AhR:Arnt/pCMV-C-HA-transfected DF-1 cells. Anti-HA was used for immunoprecipitation of the chromatin DNA fragment, the precipitated DNA was extracted and amplified by qPCR using primers spanning the AhR:Arnt binding sites. IgG was used as negative control, the input (total DNA extract) was used as positive qPCR control. The ultrapure water was used instead of the template as blank (three replicates were performed). The data are shown as means \pm SDs. Different lowercase letters represent $p<0.01,{ }^{* *} p<0.01,{ }^{*} p<0.05$, determined by ANOVA.

To further confirm that AhR:Arnt can regulate the activity of the core promoter of gga-miR-451, ChIP assay was performed to analyze whether AhR:Arnt binds to the gga-miR-451 promoter. We constructed pCMV-C-HA-AhR and pCMV-C-HA-Arnt, transfected the two vectors or empty vector (pCMV-V-HA) into DF-1 cells, and isolated chromatin for immunoprecipitation. qPCR analysis showed that a 141-bp DNA region was amplified from the anti-HA (pCMV-C-HA-AhR:Arnt) precipitates in DF-1 cells, whereas the DNA fragment was not amplified from anti-HA (pCMV-C-HA), the anti-IgG, and Mock precipitates (Figure 4C). Taken together, these results indicate that AhR:Arnt specifically binds to the gga-miR-451 promoter region and are important transcriptional regulators of gga-miR-451.

\subsection{AhR:Arnt Regulates gga-miR-451 and YWHAZ Expression}

To confirm that the AhR:Arnt could regulate gga-miR-451 expression, we transfected pcDNA3.1-AhR:Arnt (p-AhR:Arnt), pcDNA3.1, siRNA-AhR:Arnt (si-AhR:Arnt), or siRNA NC into DF-1 cells, respectively. By using qRT-PCR, AhR:Arnt overexpression significantly increased gga-miR-451 expression, whereas knockdown of AhR:Arnt decreased gga-miR-451 expression (Figure 5A). In addition, we recently reported that YWHAZ is a target gene of gga-miR-451 with negative regulation in DF-1 cells [13]. We hypothesize that AhR:Arnt can affect YWHAZ expression through regulating gga-miR-451. To test this hypothesis, Western blot analysis of identical transfected samples was performed (Figure 5B). P-AhR:Arnt markedly suppressed endogenous YWHAZ protein 
level. In contrast, si-AhR:Arnt promoted YWHAZ expression. These data suggest that AhR:Arnt binds to the core promoter of gga-miR-451, induces the expression of mature gga-miR-451, and eventually suppresses YWHAZ expression.
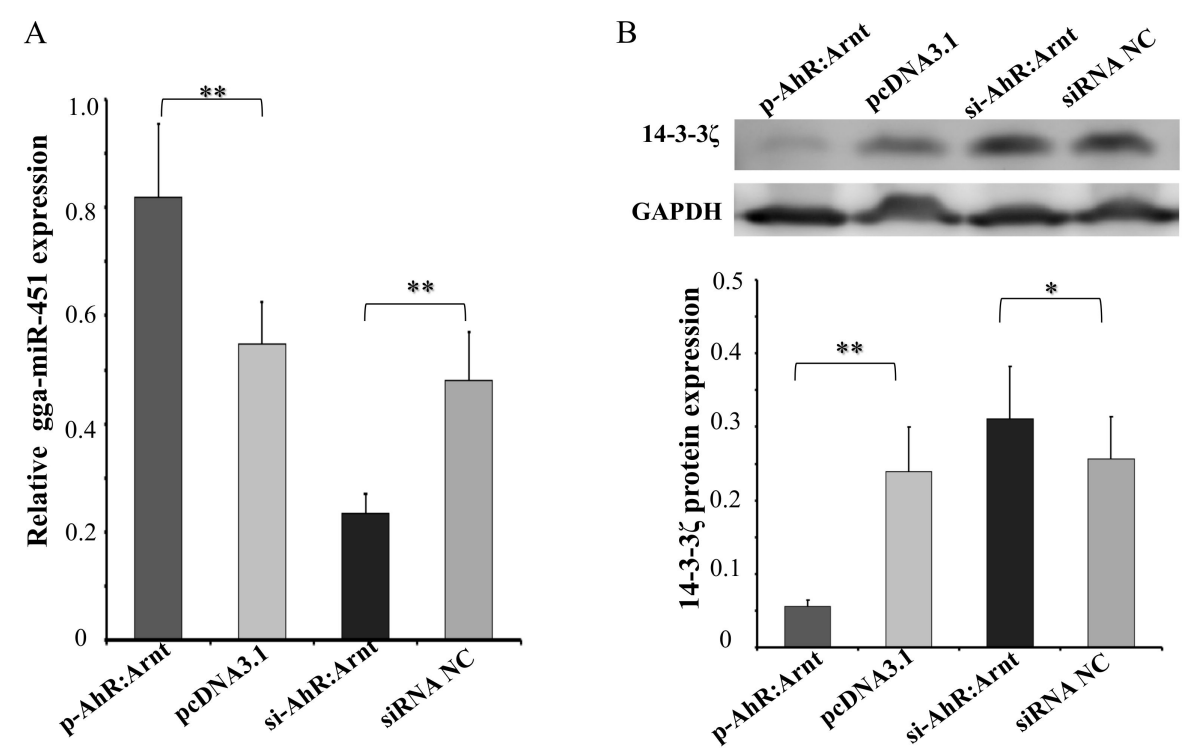

Figure 5. AhR:Arnt promotes gga-miR-451 expression decreases the expression of tyrosine3monooxygenase/tryptophan5-monooxygenase activation protein zeta (YWHAZ). (A) qRT-PCR was used to detect gga-miR-451 level $48 \mathrm{~h}$ after DF-1 cells were transfected with p-AhR:Arnt, pcDNA3.1, si-AhR:Arnt, or siRNA NC. (B) Western blot analysis was used to detect YWHAZ protein level $48 \mathrm{~h}$ post transfection with p-AhR:Arnt, pcDNA3.1, si-AhR:Arnt, or siRNA NC. The results are expressed as the means \pm SDs, with three independent replicates per group. $\left({ }^{*} p<0.05,{ }^{* *} p<0.01\right.$ ).

\subsection{MG Infection Significantly Upregulates AhR:Arnt Expression}

To further explore the role of AhR:Arnt upon MG-HS infection, we measured the expression of AhR:Arnt in MG-HS-infected DF-1 cells. RT-qPCR results showed that AhR and Arnt were significantly up-regulated in MG-infected DF-1 cells relative to its expression in non-infected DF-1 cells (Figure 6). These results suggest that AhR:Arnt plays an important role in MG infection.

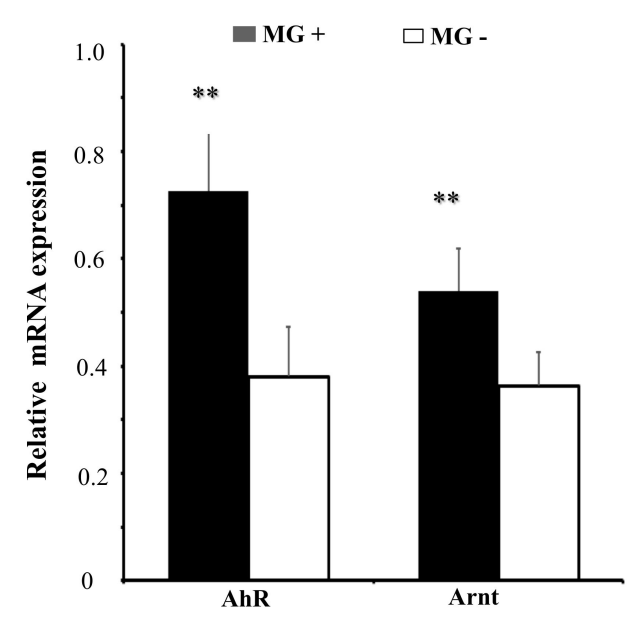

Figure 6. MG infection upregulates the expression of AhR and Arnt. DF-1 cells were infected with MG-HS as described in Materials and Methods and the total RNA was extracted. Expression of mRNA of AhR and Arnt was assessed by RT-qPCR normalized to GAPDH. The results are expressed as the means \pm SDs, with three independent replicates per group. $(* * p<0.01)$. 


\section{Discussion}

We found that miR-451 is highly conserved in the majority of vertebrate and is widely expressed in different tissues [15]. It has been found to be an important regulator of inflammation and immune responses in mammals. For example, miR-451 suppresses $\mathrm{CD}^{+} \mathrm{T}$ cell proliferative to Plasmodium parasite responses and miR-451-/- mice increased immune responses to infection [16]. Inhibition of miR-451 expression is correlated with increases in the secretion of IL-6, TNF, CCL5/RANTES, and CCL3/MIP1 $\alpha$ in mice dendritic cell [14]. In addition, miR-451 inhibits the expression of NF-kB-mediated proinflammatory molecules via inhibiting LMP7 in diabetic nephropathy [17]. We previously reported that gga-miR-451 is up-regulated in MG-infected DF-1 cells and chicken embryonic lungs with potential anti-inflammatory properties through targeting YWHAZ. gga-miR-451 inhibits the cell proliferation and cell cycle progression, and promotes cell apoptosis to facilitate MG replication [13]. Due to the important role of gga-miR-451 in MG infection, the transcriptional level regulation of the gga-miR-451 has received extensive concerns.

The biogenesis of miRNAs is a multistep and sophisticated process. They are initially transcribed into pri-miRNAs, then pri-miRNAs are processed into pre-miRNAs, and finally, into mature miRNAs [1]. The mechanism of gga-miR-451 upregulation by MG infection was studied in this article. We found the upregulation of pri-miR-451 and pre-miR-451 in MG-infected DF-1 cells, indicating that the regulation of gga-miR-451 involves transcriptional mechanisms. Interestingly, the expression of pMGA1.2 at $72 \mathrm{~h}$ post-infection was higher than its expression at 24 or 48 hpi. But pri-miR-451 and pre-miR- 451 did not follow this similar trend. This inconsistency may be due to the other complicated post-transcriptional regulation of gga-miR-451 [18]. Thus, although these data demonstrate that MG-induced gga-miR-451 was regulated transcriptionally, there remains a possibility that expression of gga-miR-451 may also be regulated at the processing level.

Most miRNAs are initially transcribed in the nucleus. Transcription factors can bind to the promoters or enhancers of miRNAs and regulate their expression. Then, miRNAs regulate the subsequent gene expression at the post-transcriptional level [19]. This may be an important element of host responses against pathogenic microorganisms infection [20]. In this study, the promoter region of gga-miR-451 gene was analyzed by the luciferase reporter assay. The data showed that the reporters PR-2174, PR-1612, and PR-386 had both foundational promoter activity and MG inducibility, but other reporters could not drive MG-induced promoter activity. It is possible that the region from -1147 to -386 may contain transcriptional repressors inhibiting MG-induced luciferase activity, whereas the region from -2174 to -1147 may contain transcriptional activators, which weaken repressors that improve MG-induced luciferase activity. However, the underlying mechanism remains to be clarified in future studies. Furthermore, functional assays of gga-miR-451 promoter revealed that transcription factor AhR:Arnt is at least partially responsible for the transcription of gga-miR-451 through three of the AhR:Arnt binding sites in DF-1 cells following MG infection. Mutant123 significantly decreased the luciferase activity of both MG-infection and non-infection compared to the full-length promoter construction (Figure 3C), whereas PR-345 did not cause decreased expression in non-infection (Figure 3B). It is possible that the PR-345 region still had two binding sites working. Direct binding of AhR:Arnt to the promoter of gga-miR-451 gene was further identified by ChIP assay. Therefore, AhR:Arnt-mediated transcriptional processing of gga-miR-451 may contribute to the upregulation by MG.

AhR is an evolutionarily conserved and ligand-activated transcription factor involved in regulation of innate immune and a variety of cellular processes, including cell proliferation, differentiation, and death [21]. Typically, cytosolic AhR activated by hydrocarbons immediately translocate to the nucleus, dimerize with Arnt and generate AhR:Arnt complex, and bind to the elements of promoter region of target genes to activate gene expression [22]. As shown in Figure 4A, overexpression of both AhR and Arnt (p-AhR:Arnt) significantly enhanced the gga-miR-451 promoter activity, compared with p-AhR or p-Arnt. This suggested that the AhR:Arnt complex worked synergistically to activate the transcriptional expression of gga-miR-451 in MG infection. Recently, many target genes were reported 
to be induced by AhR and involved in diverse biological processes including immune responses, development, and hematopoiesis $[23,24]$. For instance, AhR regulates xenobiotic detoxification through induction of cytochrome P450 1A1 (CYP1A1) and activates immune responses in the intestine [25]. AhR induced the transcription factor Notch and was crucial for IL-22-production in gut ILC22 cells [26]. Moreover, we found AhR and Arnt were up-regulated in MG-HS infection, and AhR:Arnt decreased

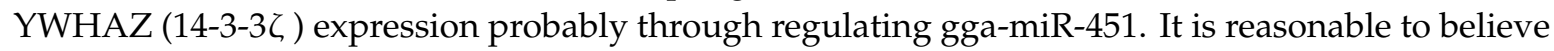
that AhR:Arnt transcription modulates gga-miR-451 expression following MG infection. In addition to AhR:Arnt, other transcription factors may also regulate miR-451 transcription. For example, c-Myc could bind on miR-451 promoter region and suppress miR-451-YWTAZ/AKT axis via recruiting HDAC3 in acute myeloid leukemia [27]. The upregulation of Paired box gene 4 (PAX4) directly regulates miR-451 expression and affects human epithelial cancer metastasis [28]. However, the molecular mechanism of these transcription factors that coordinate and regulate miR-451 gene expression needs to be further studied.

In conclusion, in the present study, we showed that the upregulation of gga-miR-451 is transcriptionally regulated in MG-infected DF-1 cells. A schematic presentation of the possible roles of AhR:Arnt in induction of gga-miR-451, as illustrated in Figure 7, showed that AhR:Arnt binds to the gga-miR-451 promoter and modulate gga-miR-451 transcription. Our discovery offers novel insight into the up-regulation of miR-451 and elucidates the mechanism of how the AhR:Arnt complex transcriptionally controls miR-451.

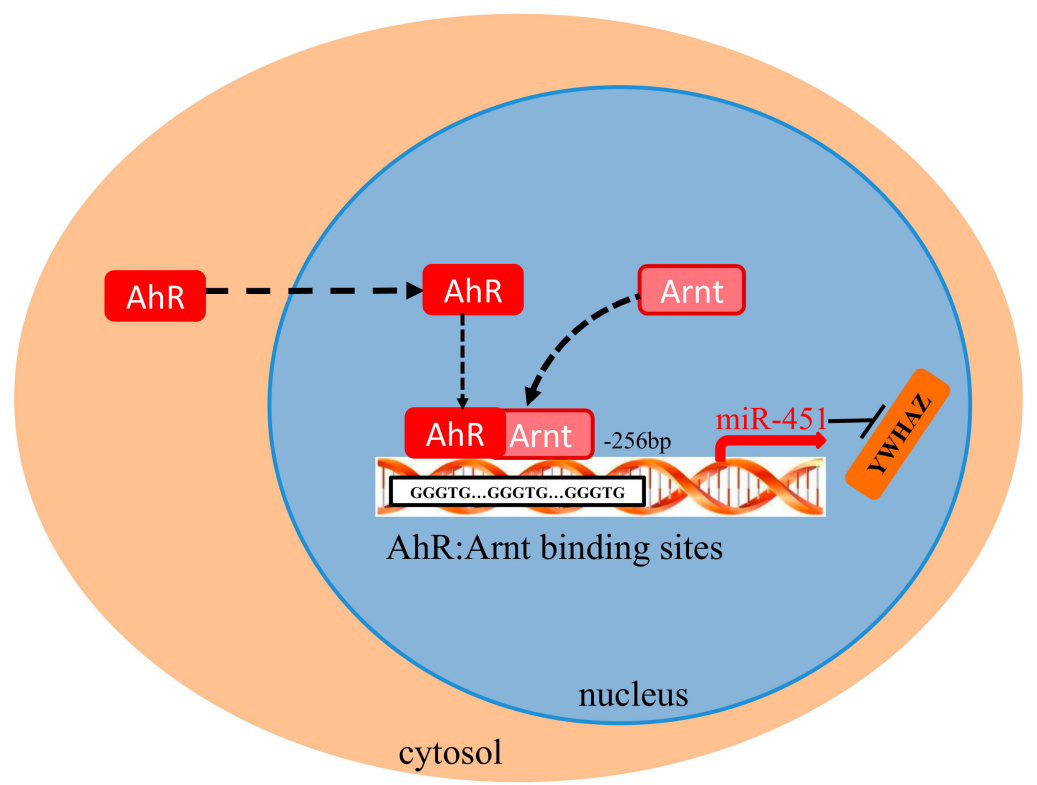

Figure 7. Graphical abstract showing the major findings of this study. Schematic summarizing the AhR:Arnt binds to the gga-miR-451 promoter and modulate gga-miR-451 transcription in DF-1 cells.

\section{Materials and Methods}

\subsection{MG-HS Culture}

The MG-HS strain used in this study is a virulent strain and donated by the State Key Laboratory of Agricultural Microbiology, College of Veterinary Medicine, Huazhong Agricultural University (Wuhan, Hubei, China). The strain was isolated from a chicken farm of Hubei Province in China [29,30]. The concentration determination of MG-HS and culture were performed as described previously. Color-changing unit (CCU) assay was used to determine the number of viable Mycoplasmas in a suspension; MG-HS at the mid-exponential phase $\left(1 \times 10^{10} \mathrm{CCU} / \mathrm{mL}\right)$ was used. 


\subsection{Cell Culture and Treatment}

The chicken embryonic fibroblast cell line (DF-1) was purchased from

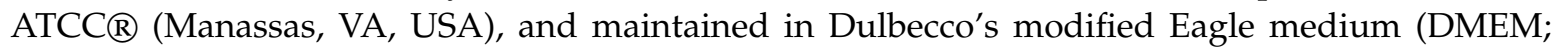
Gibco, Carlsbad, CA, USA) containing 10\% (v/v) FBS (Invitrogen, Carlsbad, CA, USA) and 1\% penicillin/streptomycin (Gibco) antibiotics. For MG-HS infection experiments, cells were plated in 6-well plates and infected with $130 \mu \mathrm{L}$ of MG-HS per well when the cells reached $\sim 80 \%$ confluence without antibiotics. For transfection experiments, cells were transfected with RNAs and/or plasmids using Lipofectamine 3000 (Invitrogen, Carlsbad, CA, USA) when the cells reached $\sim 70 \%$ confluence and were incubated for $36 \mathrm{~h}$ or $48 \mathrm{~h}$, then harvested for further use.

\subsection{Promoter Analysis}

The gene-regulation software (http://gene-regulation.com) was used to predict the transcription factor binding sites in the promoters of gga-miR-451. Potential promoters were predicted by JASPAR (http://jaspar.genereg.net).

\subsection{Plasmids Constructs}

The full length of gga-miR-451 potential promoter region and 10 deletion fragments were amplified by PCR and sub-cloned into the KpnI/HindIII sites of the pGL3-basic vector (Promega, Madison, WI, USA). Seven promoter constructs containing site-specific mutations were generated by overlap-expression PCR. To construct transcription factors AhR and Arnt expression vectors, CDS of AhR and Arnt were amplified from cDNA derived from DF-1 cells and cloned into pCDNA3.1 (p-AhR, p-Arnt). The expression vectors were double-digested with HindIII and KpnI, and then cloned into the PCMV-C-HA vector (Beyotime, Shanghai, China). The recombinant plasmids were named pCMV-C-HA-AhR and pCMV-C-HA-Arnt, respectively. The primers are described in Table 1. All plasmids were verified by sequencing.

Table 1. Sequences of DNA primers.

\begin{tabular}{|c|c|c|}
\hline Name & Primer Sequence $\left(5^{\prime}-3^{\prime}\right)$ & Accession No. \\
\hline & Primers for Cloning & \\
\hline RP 2174-F & GGGTACCCTGGCTCTTGTCC & NC_006106.5 \\
\hline PR 1612-F & GGGTACCAGCAATGCTGTGACT & \\
\hline PR 1147-F & GGGGTACCTGCTGCGATTTG & \\
\hline PR 542-F & GGGGTACCAGTCTGGGATGAAG & \\
\hline PR 386-F & GGGGTACCCATGCAGGTTCTAT & \\
\hline PR 374-F & GGGTACCTATAAAGCGGGTGCAG & \\
\hline PR 345-F & GGTACCACAAAGCAGCGGGGT & \\
\hline PR 331-F & GGTACCTCACCCCGAGAGGTG & \\
\hline PR 319-F & GGTACCCGAGAGGTGGGTGCT & \\
\hline PR 254-F & GGGGTACCACACTAACGATGCTCT & \\
\hline PR 177-F & GGGGTACCAGTTCACTATGAGACAC & \\
\hline PR R & CCCAAGCTTAACGGTTTCCCTG & \\
\hline Mut $1 \mathrm{~F}$ & GGTTCTATAAAGCATTCACAGCAGCAGCACCCCACAAA & \\
\hline Mut $1 \mathrm{R}$ & GCTGCTGCTGTGAATGCTTTATAGAACCTGCATGGCCTCATC & \\
\hline Mut $2 \mathrm{~F}$ & CCCGAGAGGTTATGTCTGCCTACGGGGACGCAGC & \\
\hline Mut $2 \mathrm{R}$ & CCGTAGGCAGACATAACCTCTCGGGGTGACCCCG & \\
\hline Mut $3 \mathrm{~F}$ & TTTGGGGACGAATGAACACTAACGATGCTCTCСТCTCСTC & \\
\hline Mut $3 \mathrm{R}$ & CATCGTTAGTGTTCATTCGTCCCCAAAGTGCCACCAC & \\
\hline pcDNA3.1-AhR F & CAAGCTGGCTAGCGTTTAAACTTAAGCTTATGAACCCCAATGTCACCTAC & XM_025146991.1 \\
\hline pcDNA3.1-AhR R & CTGGACTAGTGGATCCGAGCTCGGTACCTTACATAAATCCACTAGATGCCAAATC & \\
\hline pcDNA3.1-Arnt F & CAAGCTGGCTAGCGTTTAAACTTAAGCTTATGGCAGCCACCGCC & XM_025143392.1 \\
\hline pcDNA3.1-Arnt R & CTGGACTAGTGGATCCGAGCTCGGTACCTTACTCTGAAAAAGAAGGGAATATGT & \\
\hline pCMV-HA-AhR F & TGGCGGCCGCTCTAGCCCGGGATGAACCCCAATGTC & XM_025146991.1 \\
\hline pCMV-HA-AhR R & GCGTAATCTGGAACATCGTATGGGTATCTAGACTCGAGTTACATAAATCCACTAGATGCC & \\
\hline pCMV-HA-Arnt F & CAAGCTGGCTAGCGTTTAAACTTAAGCTTATGGCAGCCACCGCC & XM_025143392.1 \\
\hline pCMV-HA-Arnt R & CTGGACTAGTGGATCCGAGCTCGGTACCTTACTCTGAAAAAGAAGGGAATATGT & \\
\hline
\end{tabular}


Table 1. Cont.

\begin{tabular}{|c|c|c|}
\hline Name & Primer Sequence $\left(5^{\prime}-3^{\prime}\right)$ & Accession No. \\
\hline \multicolumn{3}{|l|}{ Primers for RT-qPCR } \\
\hline GAPDH-F & GAGGGTAGTGAAGGCTGCTG & NM_204305 \\
\hline GAPDH-R & CACAACACGGTTGCTGTATC & \\
\hline pMGA1.2-F & TGAAACTTCGCTCAAAGAG & AF275312 \\
\hline pMGA1.2-R & TGTAACCCAACATCATCGT & \\
\hline AhR-F & TTCCGCAGATTTCCTCCСAC & XM_025146991.1 \\
\hline AhR-R & GCTGAGCCTAAGCACAGACA & \\
\hline Arnt-F & AAACTGGAGGAGGCATCGTG & XM_025143392.1 \\
\hline Arnt-R & GTCAGCTTGTCTGGTTTGCG & \\
\hline gga-miR-451-F & GTAGGAAACCGTTACCATTACTGAG & MIMAT0003775 \\
\hline gga-miR-451-R & ACTGGTGTCGTGGAGTCGGC & \\
\hline gga-5s-rRNA-F & ССATACCACССТGGAAACGC & \\
\hline gga-5s-rRNA-R & TACTAACCGAGCCCGACCCT & \\
\hline ChIP 141bp F & CCATGCAGGTTCTATAAAGCG & NC_006106.5 \\
\hline ChIP 141bp R & GGAGAGCATCGTTAGTGTCAC & \\
\hline
\end{tabular}

The sequences of all of the primers used in this study are shown in Table 1. RNA oligonucleotides were designed and synthesized by RIBOBIO (Guangdong, China) and are shown in Table 2.

Table 2. Sequences of RNA oligonucleotides.

\begin{tabular}{cc}
\hline Name & Sequences $\left(5^{\prime}-\mathbf{3}^{\prime}\right)$ \\
\hline si-AhR & CCAACTTCCTCCAGAGAAT \\
si-Arnt & CAGACAAGCTGACCATCTT \\
\hline
\end{tabular}

\subsection{Dual-Luciferase Reporter Assay}

DF-1 cells in 24-well plates were separately co-transfected with the above plasmids (100 ng) and $10 \mathrm{ng}$ of the Renilla luciferase expression vector pRL-TK (Promega, Madison, WI, USA) using Lipofectamine 3000 (Invitrogen, Carlsbad, CA, USA) for $12 \mathrm{~h}$, and then either left uninfected or infected with $30 \mu \mathrm{L}$ of MG-HS. At $24 \mathrm{hpi}$, the cells were harvested, and the detection of luciferase activity assay was performed using the dual-luciferase assay kit (Promega, USA), according to the manufacturer's instructions. The results were normalized firefly luciferase activity to that of Renilla luciferase activity. All dual-luciferase reporter assays were performed in triplicate and repeated at least three times.

\subsection{RNA Extraction and Quantitative Real-Time (RT-qPCR)}

TRIzol reagent (Invitrogen, Carlsbad, USA) was used to extract the total RNA from treated DF-1 cells following to the manufacturer's instructions. Reverse transcription was performed using the Prime Script ${ }^{\mathrm{TM}}$ RT reagent kit with gDNA Eraser (TaKaRa, Tokyo, Japan). The expression levels of pMGA1.2, pri-miR-451, pre-miR-451, and AhR, Arnt were measured by RT-qPCR. RT-qPCR was performed on a CFX96 Touch ${ }^{\mathrm{TM}}$ instrument (Bio-Rad, USA) using TransStart Top Green qPCR SuperMix (TRANSGEN, Beijing, China). The mRNA expression levels of related genes were standardized to Glyceraldehyde-3-phosphate dehydrogenase (GAPDH) expression using the $2^{-\Delta \Delta C t}$ comparative method. The primers are listed in Table 1 . Three independent repeats were performed for all above transfection experiments.

\subsection{Chromatin Immunoprecipitation (ChIP)}

ChIP was performed using the Magna ChIP A/C kit (Millipore, catalog no. 17-10086) according to the manufacturer's instructions. In brief, $1 \times 10^{7} \mathrm{DF}-1$ cells of $10-\mathrm{cm}$ dishes were transfected with pCMV-C-HA-AhR:Arnt or pCMV-C-HA for $36 \mathrm{~h}$, and then fixed with $1 \%$ formaldehyde for $10 \mathrm{~min}$ at room temperature. Subsequently, the lysates were sonicated and then immunoprecipitated with normal anti-mouse-IgG (Millipore, St. Louis, MO, USA) as a negative control or with the antibody against HA (Abcam, Cambridge, MA, USA, ab9110); $2 \mu \mathrm{g}$ of antibody per $25 \mu \mathrm{g}$ of DNA was used. The captured chromatin was eluted and un-cross-linked by heating at $62{ }^{\circ} \mathrm{C}$, and then the immunoisolated DNA 
was subjected to qPCR amplification using primers (Table 1), TransStart Top Green qPCR SuperMix (TRANSGEN, China), and a real-time PCR detection system (Bio-Rad). The input DNA used for qPCR was equivalent to $0.01 \%$ of the original sample used for the ChIP assay.

\subsection{Western Blot Analysis}

$12 \%$ SDS-PAGE were run and transferred onto polyvinylidene difluoride (PVDF) membranes (Millipore, USA). Then, $5 \%(w / v)$ fat-free milk was used to block membranes for $1 \mathrm{~h}$ at room temperature. Primary antibodies, including YWHAZ (Cat. D155211-0025, Sangon Biotech, Shanghai, China) and GAPDH (Cat.60004-1-1g, Proteintech, Wuhan, China), were incubated at $4{ }^{\circ} \mathrm{C}$ overnight, and goat anti-rabbit secondary antibodies were incubated at room temperature for $1 \mathrm{~h}$. Washing with TBST was completed after each antibody incubation, and then the membranes were visualized with ECL ${ }^{\mathrm{TM}}$ detection system (Bio-Rad, Hercules, CA, USA).

\subsection{Statistical Analysis}

All experiments were performed in triplicate or higher. All results are presented as the mean \pm SDs. Statistical significance was determined by either ANOVA for the comparison of multiple conditions or Student's $t$-test for the comparison of two conditions, and significance was set at ${ }^{*} p<0.05,{ }^{* *} p<0.01$.

Supplementary Materials: The following are available online at http://www.mdpi.com/1422-0067/20/12/3087/s1.

Author Contributions: Y.Z. designed the study and performed the experiments; Y.F. conducted the data analysis; Y.Z., Y.S., and M.Z. drafted the manuscript; X.P. modified the manuscript. All of the authors read and approved the final manuscript for publication.

Funding: This study was supported by grants from the National Key Research and Development Program of China (2017YFD0501500), and the National Natural Science Foundation of China (Grant No. 31270216).

Conflicts of Interest: The authors declare no conflict of interest.

\section{References}

1. Gebert, L.F.R.; MacRae, I.J. Regulation of microRNA function in animals. Nat. Rev. Mol. Cell Biol. 2019, 20, 21-37. [PubMed]

2. Glazov, E.A.; Cottee, P.A.; Barris, W.C.; Moore, R.J.; Dalrymple, B.P.; Tizard, M.L. A microRNA catalog of the developing chicken embryo identified by a deep sequencing approach. Genome Res. 2008, 18, 957-964. [CrossRef] [PubMed]

3. Hu, Q.; Zhao, Y.; Wang, Z.; Hou, Y.; Bi, D.; Sun, J.; Peng, X. Chicken gga-miR-19a targets ZMYND11 and plays an important role in host defense against Mycoplasma gallisepticum (HS strain) infection. Front. Cell. Infect. Microbiol. 2016, 6, 102. [CrossRef] [PubMed]

4. Li, Z.; Chen, B.; Feng, M.; Ouyang, H.; Zheng, M.; Ye, Q.; Nie, Q.; Zhang, X. MicroRNA-23b Promotes Avian Leukosis Virus Subgroup J (ALV-J) Replication by Targeting IRF1. Sci. Rep. 2015, 5, 10294. [CrossRef] [PubMed]

5. Li, H.; Shang, H.; Shu, D.; Zhang, H.; Ji, J.; Sun, B.; Li, H.; Xie, Q. gga-miR-375 Plays a key role in tumorigenesis post subgroup J avian leukosis virus infection. PLoS ONE 2014, 9, e90878. [CrossRef] [PubMed]

6. Rodriguez, A.; Griffiths-Jones, S.; Ashurst, J.L.; Bradley, A. Identification of mammalian microRNA host genes and transcription units. Genome Res. 2004, 14, 1902-1910. [PubMed]

7. Cheng, Y.; Wei, Z.; Xie, S.; Peng, Y.; Yan, Y.; Qin, D.; Liu, S.; Xu, Y.; Li, G.; Zhang, L. Alleviation of Toxicity Caused by Overactivation of Ppar $\alpha$ through Ppar $\alpha$-Inducible miR-181a2. Mol. Ther. Nucleic Acids 2017, 9, 195-206. [CrossRef] [PubMed]

8. Zhou, J.; Lei, B.; Li, H.; Zhu, L.; Wang, L.; Tao, H.; Mei, S.; Li, F. MicroRNA-144 is regulated by CP2 and decreases COX-2 expression and PGE2 production in mouse ovarian granulosa cells. Cell Death Dis. 2017, 8 , e2597.

9. Oeckinghaus, A.; Ghosh, S. The NF-kappaB family of transcription factors and its regulation. Cold Spring Harb. Perspect. Biol. 2009, 1, a000034. [CrossRef] 
10. Winner, F.; Rosengarten, R.; Citti, C. In vitro cell invasion of Mycoplasma gallisepticum. Infect. Immun. 2000, $68,4238-4244$.

11. Markham, P.F.; Glew, M.D.; Brandon, M.R.; Walker, I.D.; Whithear, K.G. Characterization of a major hemagglutinin protein from Mycoplasma gallisepticum. Infect. Immun. 1992, 60, 3885-3891. [PubMed]

12. Chen, J.; Wang, Z.; Bi, D.; Hou, Y.; Zhao, Y.; Sun, J.; Peng, X. gga-miR-101-3p plays a key role in Mycoplasma gallisepticum (HS strain) infection of chicken. Int. J. Mol. Sci. 2015, 16, 28669-28682. [CrossRef] [PubMed]

13. Zhao, Y.; Zhang, K.; Zou, M.; Sun, Y.; Peng, X. Gga-miR-451 negatively regulates mycoplasma gallisepticum (HS strain)-induced inflammatory cytokine production via targeting YWHAZ. Int. J. Mol. Sci. 2018, 19, 1191. [CrossRef] [PubMed]

14. Weiss, M.J.; Podyminogin, R.L.; Askovich, P.S.; Navarro, G.; Rosenberger, C.M.; Zhao, G.-W.; Aderem, A. miR-451 Regulates Dendritic Cell Cytokine Responses to Influenza Infection. J. Immunol. 2012, 189, 5965-5975.

15. Xu, H.; Wang, X.; Du, Z.; Li, N. Identification of microRNAs from different tissues of chicken embryo and adult chicken. FEBS Lett. 2006, 580, 1873-3468. [CrossRef] [PubMed]

16. Chapman, L.M.; Ture, S.K.; Field, D.J.; Morrell, C.N. miR-451 limits CD4+ T cell proliferative responses to infection in mice. Immunol. Res. 2017, 65, 828-840. [CrossRef]

17. Sun, Y.; Peng, R.; Peng, H.; Liu, H.; Wen, L.; Wu, T.; Yi, H.; Li, A.; Zhang, Z. miR-451 suppresses the NF-kappaB-mediated proinflammatory molecules expression through inhibiting LMP7 in diabetic nephropathy. Mol. Cell. Endocrinol. 2016, 433, 75-86. [CrossRef]

18. Thomson, J.M.; Newman, M.; Parker, J.S.; Morin-Kensicki, E.M.; Wright, T.; Hammond, S.M. Extensive post-transcriptional regulation of microRNAs and its implications for cancer. Genes Dev. 2006, 33, 11-12. [CrossRef]

19. Liang, M.; Yao, G.; Yin, M.; Lü, M.; Tian, H.; Liu, L.; Lian, J.; Huang, X.; Sun, F. Transcriptional cooperation between p53 and NF-кB p65 regulates microRNA-224 transcription in mouse ovarian granulosa cells. Mol. Cell. Endocrinol. 2013, 370, 119-129. [CrossRef]

20. Zhou, R.; Hu, G.; Gong, A.Y.; Chen, X.M. Binding of NF-kappaB p65 subunit to the promoter elements is involved in LPS-induced transactivation of miRNA genes in human biliary epithelial cells. Nucleic Acids Res. 2010, 38, 3222-3232. [CrossRef]

21. Barouki, R.; Coumoul, X.; Fernandez-Salguero, P.M. The aryl hydrocarbon receptor, more than a xenobiotic-interacting protein. FEBS Lett. 2007, 38, 3222-3232. [CrossRef] [PubMed]

22. Øvrevik, J.; Låg, M.; Lecureur, V.; Gilot, D.; Lagadic-Gossmann, D.; Refsnes, M.; Schwarze, P.E.; Skuland, T.; Becher, R.; Holme, J.A. AhR and Arnt differentially regulate NF- $\mathrm{BB}$ signaling and chemokine responses in human bronchial epithelial cells. Cell Commun. Signal. 2014, 12, 48. [CrossRef]

23. Stockinger, B.; Meglio, P.D.; Gialitakis, M.; Duarte, J.H. The Aryl Hydrocarbon Receptor: Multitasking in the Immune System. Annu. Rev. Immunol. 2014, 32, 403-432. [CrossRef] [PubMed]

24. Singh, K.P.; Casado, F.L.; Opanashuk, L.A.; Gasiewicz, T.A. The aryl hydrocarbon receptor has a normal function in the regulation of hematopoietic and other stem/progenitor cell populations. Biochem. Pharmacol. 2009, 77, 577-587. [CrossRef] [PubMed]

25. Manzella, C.; Singhal, M.; Alrefai, W.A.; Saksena, S.; Dudeja, P.K.; Gill, R.K. Serotonin is an endogenous regulator of intestinal CYP1A1 via AhR. Sci. Rep. 2018, 8, 6103. [CrossRef] [PubMed]

26. Lee, J.S.; Cella, M.; McDonald, K.G.; Garlanda, C.; Kennedy, G.D.; Nukaya, M.; Mantovani, A.; Kopan, R.; Bradfield, C.A.; Newberry, R.D.; et al. AHR drives the development of gut ILC22 cells and postnatal lymphoid tissues via pathways dependent on and independent of Notch. Nat. Immunol. 2012, 13, 144-151. [CrossRef] [PubMed]

27. Su, R.; Gong, J.-N.; Chen, M.-T.; Song, L.; Shen, C.; Zhang, X.-H.; Yin, X.-L.; Ning, H.-M.; Liu, B.; Wang, F.; et al. c-Myc suppresses miR-451-YWTAZ/AKT axis via recruiting HDAC3 in acute myeloid leukemia. Oncotarget 2016, 7, 77430-77443. [PubMed]

28. Zhang, J.; Qin, X.; Sun, Q.; Guo, H.; Wu, X.; Xie, F.; Xu, Q.; Yan, M.; Liu, J.; Han, Z.; et al. Transcriptional control of PAX4-regulated miR-144/451 modulates metastasis by suppressing ADAMs expression. Oncogene 2015, 34, 3283-3295. [CrossRef] [PubMed] 
29. Bi, D.; Ji, X. The isolation and identification of the Mycoplasma gallisepticum. Acta Vet. Zootech. Sin 1988, $1,146-148$.

30. Bi, D.; Xu, Q. Study on pathogenicity of HS strain Mycoplasma gallisepticum. Chin. J. Anim. Poult. Infect. Dis $1997,5,24-26$.

(C) 2019 by the authors. Licensee MDPI, Basel, Switzerland. This article is an open access article distributed under the terms and conditions of the Creative Commons Attribution (CC BY) license (http://creativecommons.org/licenses/by/4.0/). 